\title{
Depatuxizumab Mafodotin
}

National Cancer Institute

\section{Source}

National Cancer Institute. Depatuxizumab Mafodotin. NCI Thesaurus. Code C105612.

An epidermal growth factor receptor (EGFR) inhibitor, with potential antineoplastic activity. Upon intravenous infusion, depatuxizumab mafodotin inhibits the activity of EGFR, thereby preventing EGFR-mediated signaling. This may inhibit tumor growth in EGFR-overexpressing tumor cells. EGFR, a receptor tyrosine kinase overexpressed in certain tumor cell types, plays a key role in tumor cell proliferation and tumor vascularization. 